\title{
iPTF13bvn: THE FIRST EVIDENCE OF A BINARY PROGENITOR FOR A TYPE Ib SUPERNOVA
}

\author{
Melina C. Bersten ${ }^{1}$, Omar G. Benvenuto ${ }^{2,3}$, Gastón Folatelli ${ }^{1}$, Ken'ichi Nomoto ${ }^{1,7}$, Hanindyo Kuncarayakti ${ }^{4,5}$, \\ Shubham Srivastav ${ }^{6}$, G. C. Anupama ${ }^{6}$, Robert Quimby ${ }^{1}$, and Devendra K. Sahu ${ }^{6}$ \\ ${ }^{1}$ Kavli Institute for the Physics and Mathematics of the Universe (WPI), The University of Tokyo, 5-1-5 Kashiwanoha, Kashiwa, \\ Chiba 277-8583, Japan; melina.bersten@ipmu.jp \\ ${ }^{2}$ Facultad de Ciencias Astronómicas y Geofísicas, Universidad Nacional de La Plata, Paseo del Bosque S/N, B1900FWA La Plata, Argentina \\ ${ }^{3}$ Instituto de Astrofísica de La Plata (IALP), CCT-CONICET-UNLP, Paseo del Bosque S/N, B1900FWA La Plata, Argentina \\ ${ }^{4}$ Millennium Institute of Astrophysics, Casilla 36-D, Santiago, Chile \\ ${ }^{5}$ Universidad de Chile, Departamento de Astronomía, Casilla 36-D, Santiago, Chile \\ ${ }^{6}$ Indian Institute of Astrophysics, Koramangala, Bangalore 560034, India \\ Received 2014 March 28; accepted 2014 July 26; published 2014 September 10
}

\begin{abstract}
The recent detection in archival Hubble Space Telescope images of an object at the location of supernova (SN) iPTF13bvn may represent the first direct evidence of the progenitor of a Type Ib SN. The object's photometry was found to be compatible with a Wolf-Rayet pre-SN star mass of $\approx 11 M_{\odot}$. However, based on hydrodynamical models, we show that the progenitor had a pre-SN mass of $\approx 3.5 M_{\odot}$ and that it could not be larger than $\approx 8 M_{\odot}$. We propose an interacting binary system as the SN progenitor and perform evolutionary calculations that are able to self-consistently explain the light curve shape, the absence of hydrogen, and the pre-SN photometry. We further discuss the range of allowed binary systems and predict that the remaining companion is a luminous O-type star of significantly lower flux in the optical than the pre-SN object. A future detection of such a star may be possible and would provide the first robust identification of a progenitor system for a Type Ib SN.
\end{abstract}

Key words: stars: evolution - supernovae: general - supernovae: individual (iPTF13bvn)

Online-only material: color figures

\section{INTRODUCTION}

An important remaining problem in astrophysics is determining the relationships between supernovae ( $\mathrm{SNe}$ ) and progenitor stars. For core-collapse SN, it is accepted that they arise from massive stars. Of particular interest is the origin of hydrogendeficient SN (Types Ib and Ic), where the mechanism to remove or deplete the outer hydrogen envelope is not well determined. The most appealing alternatives are strong stellar winds in highmass massive stars $\left(M \gtrsim 25 M_{\odot}\right)$ and mass transfer in close binary systems (see Langer 2012 for a recent review). Which of these is the dominant path for this type of SN is still unclear and the answer depends on performing detailed studies of well-observed objects.

A young Type Ib SN (He-rich, H-deficient), iPTF13bvn, was discovered by the Palomar Transient Factory on 2013 June 16 in the nearby galaxy NGC 5806. Using multi-band pre-explosion images from the Hubble Space Telescope (HST), a source was identified (within the $2 \sigma$ error box of the SN location) as the possible progenitor (Cao et al. 2013). The luminosity and colors of the progenitor candidate are consistent with some Wolf-Rayet stars (Massey et al. 2006), one of the proposed progenitors of $\mathrm{H}$-deficient SNe. Based on single stellar evolution models, Groh et al. (2013) found that a Wolf-Rayet star with zero age main sequence (ZAMS) mass of 31-35 $M_{\odot}$ was able to reproduce the observed pre-SN photometry. According to their model, at the moment of the explosion the star had a mass of $11 M_{\odot}$.

The search for progenitor stars in deep pre-explosion images is a powerful, direct approach for understanding the origin of $\mathrm{SNe}$ and it provides a critical test for stellar evolution models. Using this technique, it was possible to confirm that Type IIP $\mathrm{SNe}$ arise from the explosion of red supergiant stars (Smartt

\footnotetext{
7 Hamamatsu Professor.
}

2009). However, so far no firm progenitor identification has been reported for H-deficient SNe (Yoon et al. 2012; Groh et al. 2013; Eldridge et al. 2013). iPTF13bvn may be the first case in its class, thus allowing us, for the first time, to directly link a $\mathrm{SN} \mathrm{Ib}$ with its progenitor.

In most cases, either because the $\mathrm{SN}$ is too distant or simply lacking pre-SN images, other methods are required to infer progenitor properties. One such method is the hydrodynamical modeling of SN observations. It is a well-known fact that the morphology of the light curve (LC) is sensitive to the physical characteristics of the progenitor (e.g., see Nomoto et al. 1993; Blinnikov et al. 1998). Therefore, modeling of the LC, ideally combined with photospheric velocities or spectra, provides a useful way to constrain progenitor properties such as mass and radius, as well as explosion parameters (explosion energy and production of radioactive material). This methodology is particularly powerful when combined with stellar evolution calculations. A recent example of the predictability of this technique can be seen in our analysis of the Type IIb SN 2011dh (Bersten et al. 2012; Benvenuto et al. 2013), which allowed us to provide a self-consistent explanation of the progenitor nature that was later confirmed (Van Dyk et al. 2013; Ergon et al. 2014). Here we use the same approach to address the problem of the progenitor of iPTF13bvn.

The observational material used in this work is briefly described in Section 2. In Section 3, we present our hydrodynamical modeling of iPTF13bvn with focus on determining the mass and size of the progenitor. In Section 4, we further analyze the origin of iPTF13bvn and of the pre-explosion object, and we propose an interacting binary progenitor. The range of the allowed binary models is discussed in Section 5, where we also predict the feasibility of detecting of the companion star in future observations. In Section 6, we present the main conclusions of this work. 


\section{OBSERVATIONAL DATA}

We computed the observed bolometric LC of iPTF13bvn based on UBVRI photometry obtained by S. Srivastav et al. (2014, in preparation), and adopting bolometric corrections derived for core-collapse SNe by Lyman et al. (2014). The bolometric corrections were based on $(B-V)$ colors, although other calibrations produced compatible results. To correct colors and magnitudes to intrinsic values, we adopted a Milky Way reddening of $E(B-V)_{\mathrm{MW}}=0.0447 \mathrm{mag}$ from the NASA/IPAC Extragalactic Database (NED; Schlafly \& Finkbeiner 2011). For estimating reddening in the host galaxy, we compared the Milky Way reddening-corrected $(B-V)$ colors with an intrinsic color law derived from a sample of stripped-envelope SNe (SE SNe) observed by the Carnegie Supernova Project (M. Stritzinger et al., in preparation). The average difference with the intrinsic colors, given in the interval between the $B$-band maximum light and 20 days after, yielded a color excess of $E(B-V)_{\text {host }}=$ $0.17 \pm 0.03$ mag. We further checked this color excess with measurements of the equivalent width of the $\mathrm{Na}$ I $\mathrm{D}$ lines in the spectra of iPTF13bvn. With a measured $\mathrm{EW}_{\mathrm{NaI}} \mathrm{D}=0.5 \AA$ and adopting the relations between $\mathrm{EW}_{\mathrm{NaI}} \mathrm{D}$ and color excess given by Turatto et al. (2003), we obtained $E(B-V)=0.07$ mag or $0.22 \mathrm{mag}$, depending on the adopted relation. These values can be interpreted as an interval of valid solutions given the dispersion of the data used to derive the relations. We consider the estimate obtained from the $(B-V)$ colors to be more robust and the result to be confirmed by the $\mathrm{NaI} \mathrm{D}$ measurements. We thus adopted $E(B-V)_{\text {host }}=0.17 \pm 0.03$ mag to correct the SN observations. We employed the same value for the preSN observations under the assumption that the pre-explosion object was affected by the same amount of reddening as the $\mathrm{SN}$. This may be an underestimate of the pre-SN extinction if the explosion destroyed part of the dust that was obscuring the progenitor. Finally, to obtain luminosities, we used the average distance to NGC 5806 of $25.54 \pm 2.44 \mathrm{Mpc}$, as provided by NED. A representative uncertainty of 0.1 dex in luminosity is assumed throughout this paper. This value was estimated from the uncertainties in distance, extinction, and bolometric corrections, and summed in quadrature. The uncertainty is dominated by the systematic error in distance and is almost constant in time. The LC points may move vertically by the indicated amount, but the shape of the LC would not change significantly.

In this paper, we assumed an explosion time $\left(t_{\exp }\right)$ of JD = 2456459.24 , i.e., the mid-point between the last non-detection and the discovery date (see Cao et al. 2013). We considered an uncertainty of 0.9 days in the explosion time, based on the interval between the last non-detection and the discovery. We emphasize that this uncertainty on $t_{\exp }$ is robust due to the stringent detection limit and existing early observations in the $R$ band (see Section 3). Our bolometric LC shows a rise time to maximum of $\Delta t \approx 16$ days, i.e., near the lower end of the typical range for normal SE SNe (Richardson et al. 2006). The peak luminosity is $L_{\text {peak }} \approx 1.8 \times 10^{42} \mathrm{erg}$.

In addition to the bolometric luminosities, in Section 3 we tested our model's photospheric velocity against expansion velocities measured from the spectra. For that purpose, we adopted the Fe II expansion velocities from S. Srivastav et al. (2014, in preparation). Part of the analysis in Section 3 also aims to determine the radius of the progenitor star based on modeling the early-time LC. For this purpose, we used the $R$-band LC presented by Cao et al. (2013) because it provided the earliest coverage, starting at less than one day after discovery.

\section{HYDRODYNAMICAL MODELING}

We performed a set of explosion models using our onedimensional LTE radiation hydrodynamics code (Bersten et al. 2011). Since we were investigating an $\mathrm{H}$-free $\mathrm{SN}$, as initial structures we adopted helium stars of different masses. Specifically, we tested models with $3.3 M_{\odot}(\mathrm{HE} 3.3), 4 M_{\odot}(\mathrm{HE} 4), 5 M_{\odot}$ (HE5), and $8 M_{\odot}$ (HE8), which correspond to main-sequence masses of $12,15,18$, and $25 M_{\odot}$, respectively (Nomoto \& Hashimoto 1988). All these initial configurations have compact structures with radii of $R<3 R_{\odot}$. More details about the initial models can be found in Bersten et al. (2012).

SN parameters such as explosion energy $(E)$, ejected mass $\left(M_{\mathrm{ej}}\right)$, and the mass $\left(M_{\mathrm{Ni}}\right)$ and distribution of ${ }^{56} \mathrm{Ni}$ synthesized during the explosion were derived chiefly by analyzing the main peak of the bolometric LC and the photospheric velocity $\left(v_{\mathrm{ph}}\right)$ evolution. At first order, the evolution of $v_{\mathrm{ph}}$ is not sensitive to $M_{\mathrm{Ni}}$ and its distribution. Therefore, using the observed expansion velocity, a constraint on $E$ for each initial model (He mass) could be derived, and then $M_{\mathrm{Ni}}$ and mixing were adjusted to reproduce the bolometric LC. Figure 1 shows our hydrodynamical modeling for iPTF13bvn. While HE3.3 and HE4 can reasonably well produce the observations (LC and velocities), a slightly more massive model, HE5, already shows a worse agreement. Note that the uncertainty in the observed luminosity is nearly systematic and thus the LC could shift vertically without changing its shape. The main parameters affected by this shift are the ${ }^{56} \mathrm{Ni}$ mass and its distribution (see Figures 4 and 5 of Bersten et al. 2012). Thus, the predicted LC for model HE5 is too wide compared with the observations, regardless of any overall shift of the data points. Both HE3.3 and HE4 with similar SN parameters appear to be equally plausible. We thus consider our best solution to be that with intermediate parameters between those models. That means that iPTF13bvn was produced by the explosion of a low-mass He star of $\approx 3.5 M_{\odot}$, with an ejected mass of $M_{\text {ej }} \approx 2.3 M_{\odot}{ }^{8}$, an explosion energy of $E=7 \times 10^{50}$ erg and a ${ }^{56} \mathrm{Ni}$ yield of $M_{\mathrm{Ni}} \approx 0.1 M_{\odot}$. These parameters indicate that iPTF13bvn was a low-energy event with normal nickel production. Also, we found that a quite strong mixing (extending to $\approx 96 \%$ of the initial mass) was required in all the calculations to reproduce the rise time of the LC, which in turn is very well known due to the strong constraint on the explosion time ( $t_{\mathrm{exp}}$; Cao et al. 2013).

The low progenitor mass suggested by our modeling is in clear contradiction with the range of masses allowed for Wolf-Rayet stars (e.g., see Heger et al. 2003; Groh et al. 2013), and thus our results are in disagreement with those of Groh et al. (2013). Specifically, in Figure 1 we also show the case of a He star with $8 M_{\odot}$ for an explosion energy of $3 \times 10^{51} \mathrm{erg}$. We show a compromise solution for this model that produces velocities too high and an LC too wide. If we adopted a lower value of the explosion energy in order to better fit $v_{\mathrm{ph}}$, then the LC would become even fainter and wider, in worse agreement with the observations. Note also that although the HE8 model shown in Figure 1 could be made more compatible with the observations by shifting the explosion date to about one week earlier, that would be incompatible with the non-detection limit in the $R$ band that is shown in Figure 2. Even considering all the uncertainties related with the model hypotheses and with the observations,

\footnotetext{
${ }^{8} \quad M_{\mathrm{ej}}=M_{\mathrm{tot}}-M_{\text {cut }}$, where $M_{\mathrm{tot}}$ is the total mass of the He star and $M_{\text {cut }}$ is
} the mass assumed to form a compact remnant. 

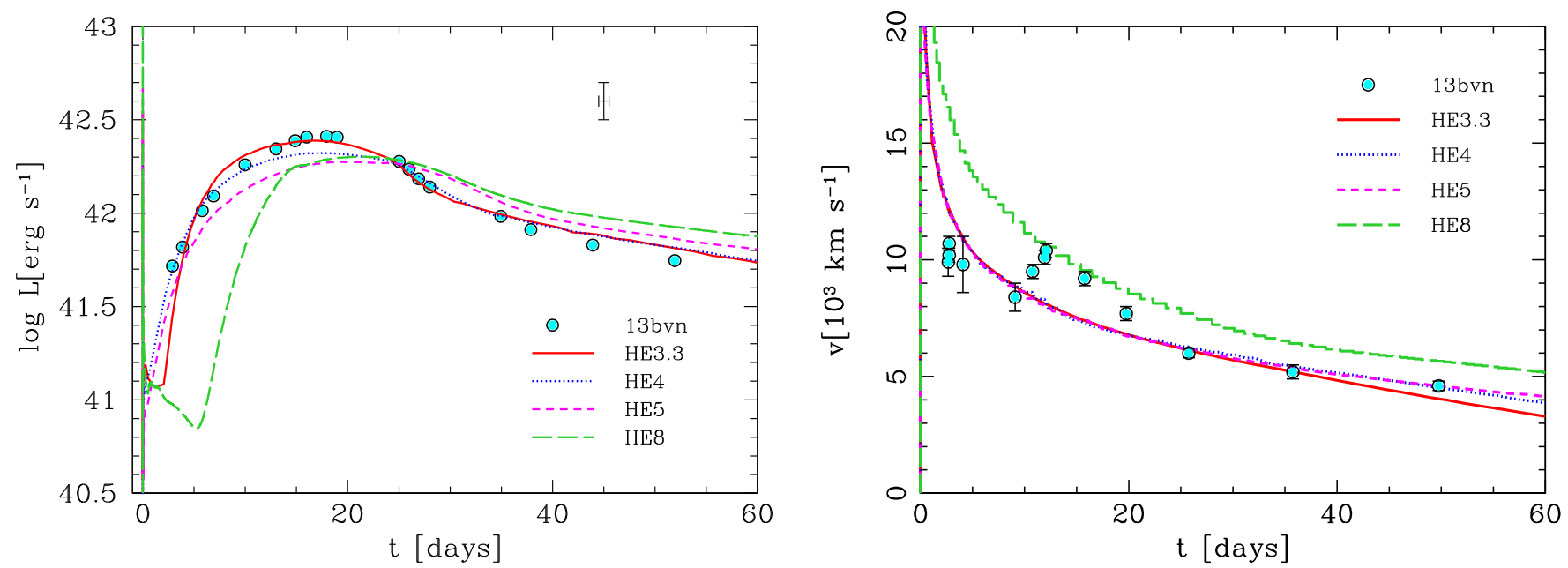

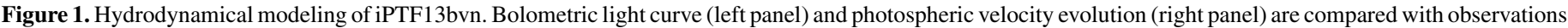

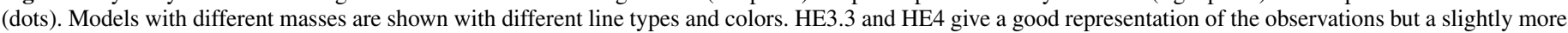

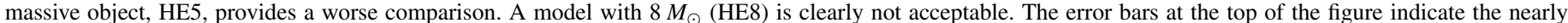
constant uncertainty in luminosity and the adopted uncertainty in the explosion time (see Section 2).

(A color version of this figure is available in the online journal.)

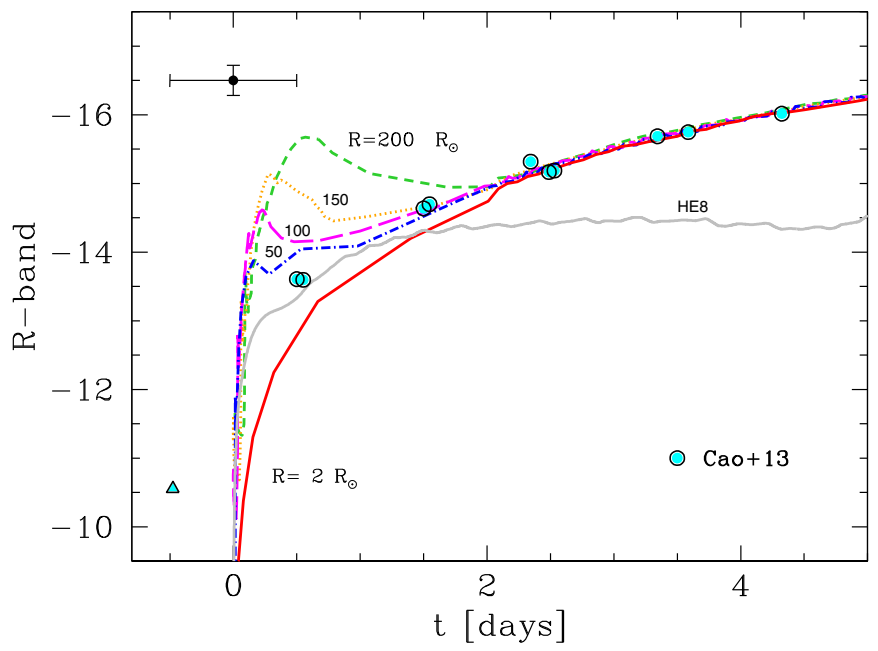

Figure 2. Early $R$-band light curve of iPTF13bvn (dots) compared with models of different progenitor radii (lines). The labels next to the curves indicate the radius in $R_{\odot}$. The radius variation is accomplished by attaching essentially massless $\left(<0.01 M_{\odot}\right)$ envelopes to the compact He-star model HE4 (see Section 3). In spite of the good constraint on $t_{\text {exp }}$, its uncertainty (black line) is still too large to distinguish between a compact star (of a few $R_{\odot}$ ) and a relatively extended structure (of $R \lesssim 150 R_{\odot}$ ). A better constraint on $t_{\exp }$ or a higher cadence of the observations is required to capture the short-duration emission feature produced by relatively extended progenitors. The error bars indicate the size of the uncertainty in magnitude (dominated by the distance uncertainty) and in the explosion time (see Section 2). Model HE8 is included to show that it would not be compatible with a shift in the explosion date of more than $\approx 1$ day.

(A color version of this figure is available in the online journal.)

we can firmly rule out models with He core mass $\gtrsim 8 M_{\odot}$ as progenitors of iPTF13bvn.

The compactness of the progenitor can be explored if the SN is observed early enough, before its emission becomes powered by ${ }^{56} \mathrm{Ni}$ decay. The shape of the LC in this early phase is given by the progenitor size and, to some extent, by the degree of ${ }^{56} \mathrm{Ni}$ mixing. The good constraint on $t_{\exp }$ and the absence of an initial peak in the early LC of iPTF13bvn can lead to the naive conclusion that the progenitor should have been a compact star of a few solar radii in size. We have tested this by attaching thin He-rich envelopes of different radii to our HE4 model, as described in Bersten et al. (2012). ${ }^{9}$ We derive $R$-band photometry from the models by assuming a blackbody spectral energy distribution (SED). Figure 2 shows the early $R$-band LC compared with our models. From the comparison, it is clear that models with relatively extended structures, $R \lesssim 150 R_{\odot}$, cannot be ruled out considering the uncertainty in $t_{\exp }(\approx 0.9$ days $)$. Therefore, the progenitor of iPTF13bvn is not necessarily a compact star. Interestingly, our modeling suggests that in order to capture the differences in LC shapes and thus to discriminate between compact and relatively extended progenitors, it is necessary to obtain several observations during the same night.

\section{BINARY PROGENITOR}

The mass we derived from hydrodynamical modeling is difficult to reconcile with the idea of a single progenitor for the Type Ib SN iPTF13bvn. In order to remove the hydrogen envelope, a single star should be massive enough $\left(M_{\text {ZAMS }} \gtrsim\right.$ $\left.25 M_{\odot}\right)$ to produce strong stellar winds that are able to strip the envelope during its evolution (see, e.g., Langer 2012). However, in that case, the resulting helium star mass would be too large $\left(M_{\mathrm{He}} \gtrsim 8 M_{\odot}\right)$ to account for the observed SN LC, as shown in Section 3. Alternatively, the path to the explosion of a low-mass $\mathrm{He}$ star is naturally provided by interacting binaries. Although Cao et al. (2013) derived a mass-loss rate that is compatible with a massive Wolf-Rayet star based on radio observations, this result depends strongly on the assumed wind velocity among other parameters that are not completely known. For a lowmass helium star, a lower mass-loss rate is expected but the wind properties are uncertain; therefore, the radio observations cannot be used to validate or reject this possibility.

The question is whether or not there are binary configurations capable of simultaneously reproducing the $\mathrm{SN}$ properties and the pre-explosion photometry.

To address this question, we used the binary stellar evolutionary code employed in our analysis of SN 2011dh (Benvenuto et al. 2013) which is a recent update of the code presented by

\footnotetext{
9 Note that low-mass HE stars can experience an expansion of their outer envelope, as shown by Yoon et al. (2010; see also Section 4).
} 


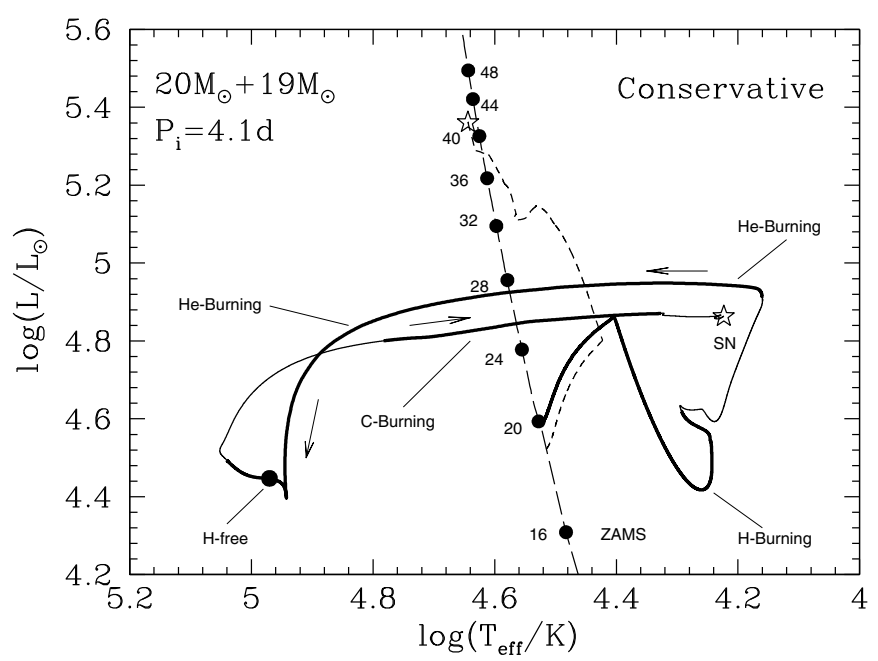

Figure 3. Evolutionary tracks of the binary components of the progenitor of iPTF13bvn for a proposed system with initial masses of $20 M_{\odot}$ and $19 M_{\odot}$ and an initial orbital period of 4.1 days. The solid line indicates the track of the primary (donor) star (arrows show the evolutionary progress). The short-dashed line shows the evolution of the secondary (accretor) star. Fully conservative accretion $(\beta=1)$ is assumed. The star symbols show the location of both components at the moment of explosion of the primary star. Thick portions of the primary's track indicate the phases of nuclear burning at the stellar core. The long-dashed line shows the locus of the ZAMS, with dots showing different stellar masses (labels in units of $M_{\odot}$ ).

Benvenuto \& De Vito (2003). For the present problem, we considered non-rotating stars. At mass transfer conditions, the code simultaneously solves the donor star structure, the mass transfer rate, and the orbital evolution in a fully implicit way, providing numerical stability (see Benvenuto \& De Vito 2003). Figure 3 shows the evolutionary tracks in the $\mathrm{H}-\mathrm{R}$ diagram for a system composed by a donor (primary) star of $20 M_{\odot}$ and an accretor (secondary) star of $19 M_{\odot}$ on a circular orbit with an initial period of 4.1 days. We further assumed conservative mass transfer, i.e., that all the mass that is transferred is accreted onto the secondary (parameterized by the accretion efficiency, $\beta=1$ ). The choice of the initial masses and orbital period of the system was guided to account for the composition of the exploding star, the $\mathrm{LC}$ of the SN, and the available photometric observations prior to the explosion. Such configuration is by no means unique, but it serves to demonstrate the feasibility of the binary progenitor scenario. We will explore the range of possible progenitor systems in greater detail in Section 5.

The system undergoes class A mass transfer-i.e., it experiences the first Roche-lobe overflow (RLOF) when it is still in the core hydrogen burning stage. It detaches shortly after core hydrogen exhaustion and, as consequence of the formation of a shell burning hydrogen, suffers a second RLOF episode until core helium ignition. After detachment, the donor star evolves blueward up to very high effective temperatures. At these stages, the star undergoes wind mass loss that removes the hydrogenrich outer layers. The mass loss has been treated as in Yoon et al. (2010), setting the correction factor for Wolf-Rayet winds defined in their Equation (1), $f_{\mathrm{WR}}$, to 2.5.

After helium core exhaustion, the donor star evolves redward reaching the final pre-SN structure. We stopped the calculation at oxygen core exhaustion, assuming that no significant displacement in the H-R diagram occurs until core collapse. In the meantime, the companion star accretes hydrogen-rich material, which causes it to brighten. As the accretion rate is rather low, this star moves upward in the H-R diagram without swelling ap-

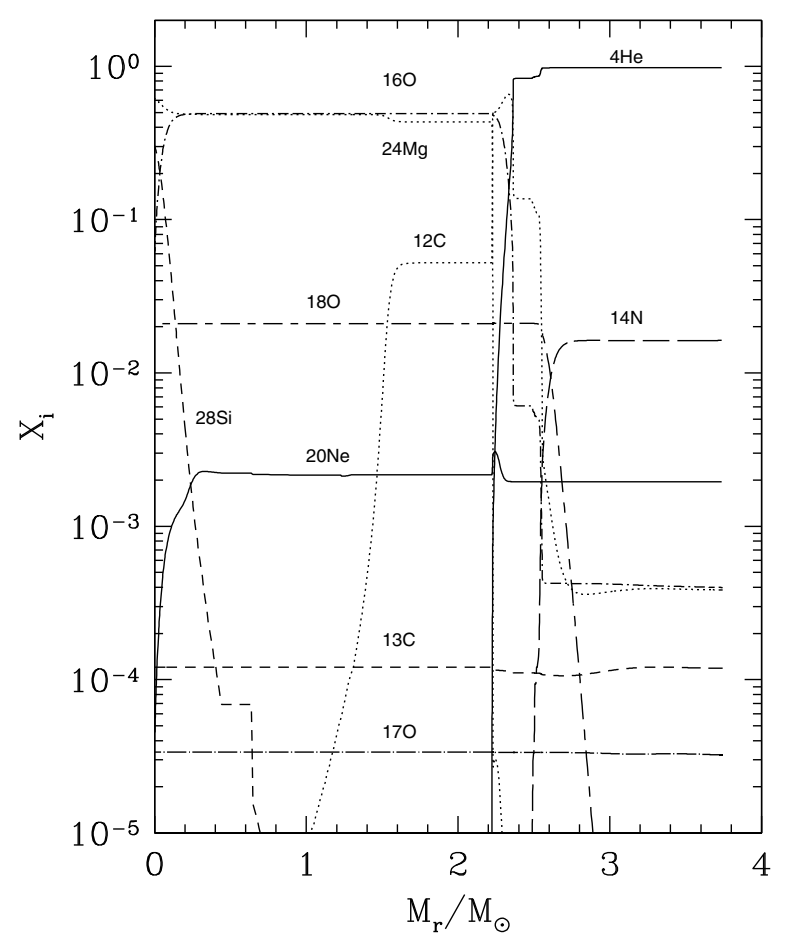

Figure 4. Chemical structure of the primary star near to oxygen core exhaustion. At this stage the star is already devoid of hydrogen.

preciably. In this way, the system avoids undergoing a common envelope episode.

At the time of the explosion the primary is an H-free star (see Figure 4 ) with a mass of $3.74 M_{\odot}$ and a radius of $32.3 R_{\odot}$, in concordance with our hydrodynamical estimations. The companion star reaches a mass of $33.7 M_{\odot}$, with luminosity and effective temperature $\left[\log \left(L / L_{\odot}\right)=5.36\right.$ and $\log \left(T_{\text {eff }} / \mathrm{K}\right)=$ 4.64] comparable to those corresponding to a ZAMS star of $\approx 42 M_{\odot}$. The companion star is thus appreciably overluminous, in agreement with previous predictions (see, e.g., Dray \& Tout 2007).

Figure 5 shows that the pre-SN state of the proposed binary progenitor is compatible with the HST observations. To perform this comparison, we assumed the primary's SED is well reproduced by a blackbody of the given temperature and luminosity. This is a reasonable approximation for a low-mass $\mathrm{He}$ star, as suggested by Yoon et al. (2012). For the secondary star, we adopted an atmosphere model from Kurucz (1993) for a main-sequence star of the corresponding effective temperature, scaled to reproduce the required luminosity. We summed both contributions, applied the extinction correction derived in Section 2, and converted to observed flux adopting the distance of $25.5 \mathrm{Mpc}$. The synthetic photometry of the progenitor system in the three existing bands is in agreement with the observations within the uncertainties, with differences of less than 0.1 mag. We note that the primary star dominates the flux in the optical regime, so its explosion should eventually leave a much fainter object (i.e., the companion star). ${ }^{10}$ The disappearance of the primary star could be confirmed once the SN fades below its brightness. Considering the usual decline rates of SE SNe, we estimate to occur at about $3 \mathrm{yr}$ after the explosion.

\footnotetext{
10 Even if the secondary is bolometrically more luminous than the primary
} star, its emission in the optical range is lower due to its hotter temperature. 


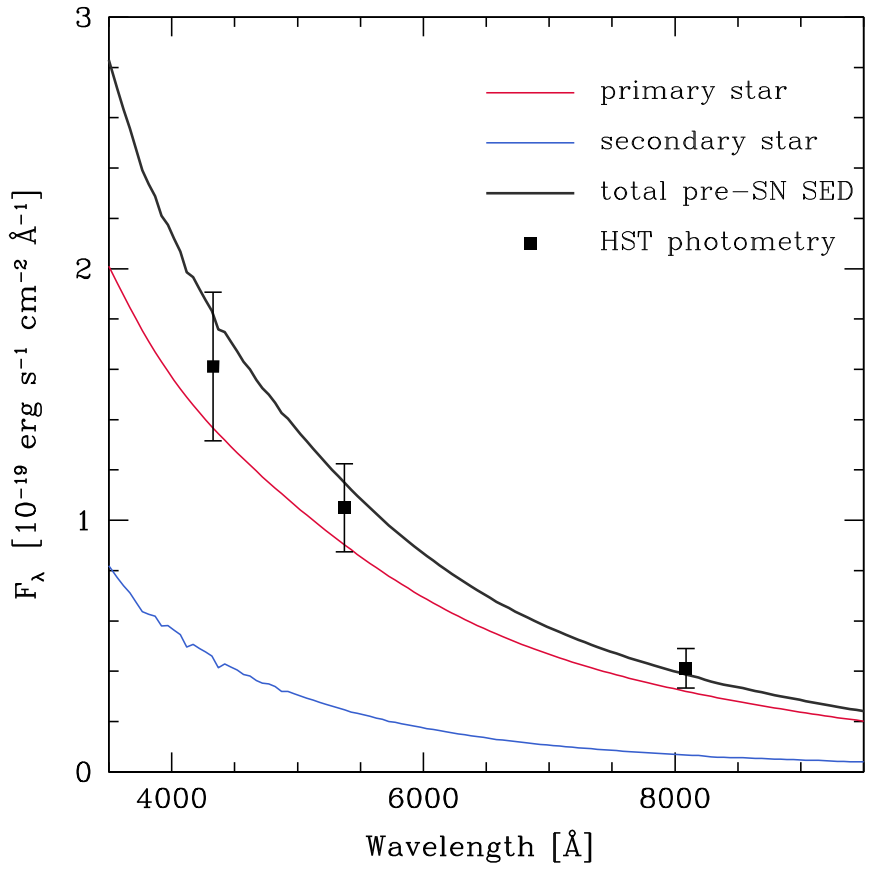

Figure 5. Predicted spectrum of the binary progenitor (solid black line) compared with HST pre-SN photometry (black squares). The binary spectrum is the sum of a primary star approximated by a blackbody (red line) and a secondary star represented by an atmosphere model of Kurucz (1993; blue line). The spectra have been extinguished assuming a standard reddening law (Cardelli et al. 1989) and adopting the extinction value derived in Section 2. The HST photometry was adopted from Cao et al. (2013) and converted to specific fluxes at the approximate effective wavelength of the $F 435 \mathrm{~W}, F 555 \mathrm{~W}$, and $F 814 \mathrm{~W}$ bands.

(A color version of this figure is available in the online journal.)

\section{PREDICTABILITY OF THE COMPANION STAR}

The solution to the progenitor system presented in Section 4 is not unique. Based on the pre-explosion photometry and the SN observations, we studied the range of allowed binary systems with the aim of predicting the nature of the remaining companion star. The following analysis is not intended to be an accurate derivation but an approximation based on our calculations and general knowledge of interacting binaries.

The first condition for the binary scenario is that the pre-SN structure should have a mass compatible with the LC and should be devoid of hydrogen. A primary star initially more massive than $\approx 25 M_{\odot}$ would result in a helium core too massive to reproduce the SN LC. On the opposite extreme, if the initial mass of the primary were $\lesssim 15 M_{\odot}$, it would be difficult to find hydrogen-free structures. Furthermore, the range of allowed pre-explosion masses for the helium star (3-5 $M_{\odot}$ ) places some limits on its final luminosity. Following Yoon et al. (2010, 2012), we consider this range to be roughly $4.6 \lesssim \log \left(L_{1}^{\mathrm{f}} / L_{\odot}\right) \lesssim 5.0$.

The secondary star, in turn, should not have a mass too close to that of the primary to prevent it from evolving before the explosion (Claeys et al. 2011; Benvenuto et al. 2013). If it had evolved, and considering the allowed range of luminosity of the primary, the system luminosity would have become too large. This ensures that the companion star should remain near the ZAMS. Its exact location on the H-R diagram depends on its final mass, which in turn is determined by the total mass of the system and the accretion efficiency, $\beta$. In order to avoid common envelope episodes, which is beyond the capabilities of the present code, we have additionally required that the mass ratio of the system was not lower than $M_{2}^{\mathrm{i}} / M_{1}^{\mathrm{i}}=0.8^{11}$.

The above restrictions allowed us to place constraints on the final mass-and thereby luminosity — of the companion star. Considering approximate ranges of initial primary mass of $15 \lesssim$ $M_{1}^{\mathrm{i}} \lesssim 25 M_{\odot}$ and initial mass ratio of $0.8 \lesssim M_{2}^{\mathrm{i}} / M_{1}^{\mathrm{i}} \lesssim 0.95$, the final secondary mass results in the range of $23 \lesssim M_{2}^{\mathrm{f}} \lesssim 45 M_{\odot}$. Given the uncertainties on the mass accretion mechanism, we relaxed the condition on $\beta$ to be $0.5 \lesssim \beta \leqslant 1$, and we thus found that $18 \lesssim M_{2}^{\mathrm{f}} \lesssim 45$. Additionally, the secondary star is expected to be overluminous compared to normal ZAMS stars of equal mass, as we found for the system presented in Section 4. Its luminosity would correspond to that of a normal ZAMS star of $\approx 20 \%$ larger mass (see Figure 3 ). This implies a range of luminosities on the ZAMS of $4.6 \lesssim \log \left(L_{2}^{\mathrm{f}} / L_{\odot}\right) \lesssim 5.6$. We corroborated that such a range was not further reduced by the constraints from the pre-explosion photometry.

This analysis suggests that the explosion of iPTF13bvn has left a remnant companion star of O-type characteristics. We thus predict that this object could be recovered with future deep observations once the light from the SN ejecta becomes faint enough. With the currently available instrumentation of $H S T$, secondary stars with $\log \left(L / L_{\odot}\right) \gtrsim 5.3$ will be detectable at signal-to-noise ratio $(\mathrm{S} / \mathrm{N}) \gtrsim 5$ with exposures times of the order of one hour in the near-UV and blue optical bands.

We finally note that the HST pre-explosion photometry is in principle also compatible with a single underluminous (by a factor of 1.5-2) late B- or early A-type supergiant star. Such object can be discarded as the progenitor of a SE SN. In order not to affect the photometry, the actual progenitor should be at least one order of magnitude less luminous in the optical range than the supergiant. One possibility would be a Wolf-Rayet star, but that is ruled out by our hydrodynamical analysis. The alternative is that the $\mathrm{SN}$ was produced by the merger of two unseen compact stars. Such an alternative can be tested once the SN light fades from sight by checking whether the flux of the remaining object has remained nearly unchanged.

\section{CONCLUSIONS}

Our hydrodynamical analysis of iPTF13bvn pointed to the explosion of a low-mass helium star (of $\approx 3.5 M_{\odot}$ ) with a relatively low explosion energy (of $\approx 7 \times 10^{50} \mathrm{erg}$ ) and normal production of radioactive material $\left(M_{\mathrm{Ni}} \approx 0.1 M_{\odot}\right)$. Interestingly, from the LC rise time we could conclude that this relatively normal event managed to produce a quite strong ${ }^{56} \mathrm{Ni}$ mixing. Our conclusion about the low pre-SN mass is robust because of the strong constraint on the explosion time and it is not affected by the systematic uncertainty of 0.1 dex in luminosity.

Our LC modeling is in contradiction with a Wolf-Rayet progenitor for iPTF13bvn, as suggested by Groh et al. (2013). In order to explain the explosion of a low-mass helium star, we proposed the possibility of an interacting binary progenitor. We showed that a system composed of $20 M_{\odot}+19 M_{\odot}$ stars and an initial orbital period of 4.1 days can fully satisfy all the observational constraints (pre-explosion mass, chemical composition, and HST photometry). The primary star is expected to dominate the flux of the progenitor in the optical, so, as a result of the SN explosion, we predict that the flux in the observed bands will decrease significantly when the SN fades.

\footnotetext{
${ }^{11}$ Note that this constraint is not physically motivated. Therefore, it may affect our predictability at the low end of the secondary luminosity.
} 
We went one step beyond and studied the possible binary configurations that could lead to compatible solutions for all the observational requirements with the focus on making predictions about the putative companion star. We found that the remaining star should necessarily be close to the ZAMS with a range of luminosities of $4.6 \lesssim \log \left(L_{2}^{\mathrm{f}} / L_{\odot}\right) \lesssim 5.6$ This means that the companion star may be detected in the future with deep HST imaging in the UV-blue range. The detection of the companion would produce the first robust identification of a hydrogen-deficient SN progenitor as a binary system.

While recent evidence suggests a large fraction of massive stars belong to interacting binary systems (Sana et al. 2012), it is still not clear whether this is the main channel to produce hydrogen-free SN. The combination of hydrodynamical SN models and close binary evolution calculations proves to be a powerful tool for understanding the nature of these events in a self-consistent way.

Finally, we studied the implications on the progenitor size by modeling the early $R$-band LC. Contrary to what might be expected from its monotonic rise, we showed that compact structures (of a few $R_{\odot}$ ) as well as relatively extended envelopes $\left(\lesssim 150 R_{\odot}\right)$ are allowed with the present cadence of the observations. Our calculations suggest that sub-night cadence is required to distinguish among progenitor sizes in the above range. Ongoing surveys such as the Kiso Supernova Survey or future programs like the intermediate Palomar Transient Factory and the Zwicky Transient Facility will be able to provide the necessary frequency of observations to solve this kind of problem.

We thank A. Gal-Yam and Y. Cao for kindly providing earlytime data. This research has been supported in part by the Grant-in-Aid for Scientific Research of MEXT (22012003 and 23105705) and JSPS (23540262) and by the World Premier International Research Center Initiative, MEXT, Japan. O.G.B. is Member of the Carrera del Investigador Científico of the Comisión de Investigaciones Científicas (CIC) of the Provincia de Buenos Aires, Argentina. G.F. acknowledges financial support by a Grant-in-Aid for Scientific Research for Young Scientists (23740175). G.C.A. and D.K.S. acknowledge partial support from DST under the India-Japan S\&T Cooperation programs. Support for H.K. is provided by the Ministry of Economy, Development, and Tourism's Millennium Science Initiative through grant IC12009, awarded to The Millennium Institute of Astrophysics, MAS. H.K. acknowledges support by CONICYT through FONDECYT grant 3140563.

\section{REFERENCES}

Benvenuto, O. G., Bersten, M. C., \& Nomoto, K. 2013, ApJ, 762, 74 Benvenuto, O. G., \& De Vito, M. A. 2003, MNRAS, 342, 50

Bersten, M. C., Benvenuto, O., \& Hamuy, M. 2011, ApJ, 729, 61

Bersten, M. C., Benvenuto, O. G., Nomoto, K., et al. 2012, ApJ, 757, 31

Blinnikov, S. I., Eastman, R., Bartunov, O. S., Popolitov, V. A., \& Woosley, S. E. 1998, ApJ, 496, 454

Cao, Y., Kasliwal, M. M., Arcavi, I., et al. 2013, ApJL, 775, L7

Cardelli, J. A., Clayton, G. C., \& Mathis, J. S. 1989, ApJ, 345, 245

Claeys, J. S. W., de Mink, S. E., Pols, O. R., Eldridge, J. J., \& Baes, M. 2011, A\&A, 528, A131

Dray, L. M., \& Tout, C. A. 2007, MNRAS, 376, 61

Eldridge, J. J., Fraser, M., Smartt, S. J., Maund, J. R., \& Crockett, R. M. 2013, MNRAS, 436, 774

Ergon, M., Sollerman, J., Fraser, M., et al. 2014, A\&A, 562, A17

Groh, J. H., Georgy, C., \& Ekström, S. 2013, A\&A, 558, L1

Groh, J. H., Meynet, G., Georgy, C., \& Ekström, S. 2013, A\&A, 558, A131

Heger, A., Fryer, C. L., Woosley, S. E., Langer, N., \& Hartmann, D. H. 2003, ApJ, 591,288

Kurucz, R. 1993, ATLAS9 Stellar Atmosphere Programs and 2 km/s Grid, Kurucz CD-ROM (Cambridge, MA: Smithsonian Astrophysical Observatory), 13

Langer, N. 2012, ARA\&A, 50, 107

Lyman, J. D., Bersier, D., \& James, P. A. 2014, MNRAS, 437, 3848

Massey, P., Olsen, K. A. G., Hodge, P. W., et al. 2006, AJ, 131, 2478

Nomoto, K., \& Hashimoto, M. 1988, PhR, 163, 13

Nomoto, K., Suzuki, T., Shigeyama, T., et al. 1993, Natur, 364, 507

Richardson, D., Branch, D., \& Baron, E. 2006, AJ, 131, 2233

Sana, H., de Mink, S. E., de Koter, A., et al. 2012, Sci, 337, 444

Schlafly, E. F., \& Finkbeiner, D. P. 2011, ApJ, 737, 103

Smartt, S. J. 2009, ARA\&A, 47, 63

Turatto, M., Benetti, S., \& Cappellaro, E. 2003, in Proc. ESO/MPA/MPE Workshop, From Twilight to Highlight: The Physics of Supernovae, ed. W. Hillebrandt \& B. Leibundgut (Berlin: Springer), 200

Van Dyk, S. D., Zheng, W., Clubb, K. I., et al. 2013, ApJL, 772, L32

Yoon, S.-C., Gräfener, G., Vink, J. S., Kozyreva, A., \& Izzard, R. G. 2012, A\&A, $544, \mathrm{~L} 11$

Yoon, S.-C., Woosley, S. E., \& Langer, N. 2010, ApJ, 725, 940 